\title{
Simultaneous Parameters Identification and State Estimation based on Unknown Input Observer for a class of LPV Systems.
}

\author{
M. Fouka, L. Nehaoua, H. Arioui and S. Mammar
}

\begin{abstract}
A novel unknown input observer structure for parameters and state estimation is proposed to enhance the performance of the estimator. In this paper, we suggest how a failed matching condition in a nonlinear unknown input observer can be recovered by using time delayed measurement to solve the inversing problem. Based on delayed outputs, an augmented system is constructed from which the parameters of the model and the system states can be simultaneously estimated. The augmented nonlinear model is transformed into a Takagi Sugeno (TS) form.

Sufficient conditions for the existence of the estimator are given in terms of linear matrix inequalities (LMIs). Using the obtained information on the unknown input observer, unknown parameters are identified. Finally, the feasibility and the effectiveness of the suggested approach is demonstrated on examples.
\end{abstract}

\section{INTRODUCTION}

$\mathrm{E}$ STIMATION is a common approach to deal with many problems occuring in various application areas: in motion control design, signal processing, process identification and fault detection. First theory about the state estimation was introduced in the sixties of the previous century by Luenberger for deterministic linear systems [1]. After this original work, many other approaches have been proposed for both the linear and the nonlinear cases. Among them, one can cite sliding mode observer [2], extended Kalman observer [3]. Nevertheless, a particular attention was paid for the nonlinear estimation which has been the subject of an intensive research during the last decades. However, several challenges need more thorough investigation.

The first challenge was to consider the system environment in observer design process. Indeed, any system has inputs and outputs but also it may be subject to various intrinsic parameter uncertainties, external disturbances and, unknown input. So, it is a common practice to simultaneously reconstruct the systems states and unknown inputs. This latter issue has become a challenging problem that received a particular attention. Wang [4] proposes a procedure to design a reduced-order observers without any knowledge about the unknown inputs. Darouach [5] has presented a simple method to design a full-order observer for a linear system with unknown inputs where the existence of the observer is guaranteed by a set of necessary and sufficient conditions. For diagnosis application, Koenig [6] considers a Proportional-Integral observer form to estimate simultaneously the systems states and the fault signals. Afterwards, the

All the authors are with the IBISC Laboratory, Evry-Val-d'Essonne University, 40, rue de Pelvoux, 91020 Evry Courcouronne Cedex Majda-Amina.Fouka@ufrst. univ-evry.fr second challenge was to deal with systems parameter variations and nonlinearities. Many works have been proposed to design UIO by considering the direct nonlinear approach [7]. Beyond the previous considerations, a forward solution was the use of the linear system theory and numeric optimization tools to facilitate the UIO design procedure. In this sense, an extensive literature can be found about the UIO design by using Linear Parameter Variation techniques (LPV) [8] and Takagi-Sugeno models (TS) [9]. Indeed, the use of TS models with measurable premise variables allows to describe a nonlinear system with a good precision [10]. Even in [12], Ichalal and al. discuss a simultaneous state and unknown inputs estimation for nonlinear systems described by a TS model with unmeasurable premise variables.

Until now, the discussion above concerns the simultaneous state and unknown inputs estimation. At our best knowledge, the simultaneous parameters and state estimation with an unknown input observer has never been addressed yet. Nonetheless, Kuhlmann, in [11], studied the feasibility of the parameter and state estimation of nonlinear systems by using a multi-observer technique to be used in a supervisory framework. This work consists to design a hybrid observer starting from a set of nominal parameters and, a switch condition to select one of the resulting observers. However, the problem formulation is complex even for a linear system case, and the switch condition is based on a preliminary probabilistic knowledge of the current parameters within the parameter set.

In this paper, we propose a generalization of the UI observers for nonlinear systems. In the present work, nonlinearities are transformed into a TS exact form [13] which is used to simultaneously estimate the unknown inputs, the models parameters and, the systems states. We also describe our contributions to solve the observer design problem where the matching condition is initially not fulfilled. For this, delayed outputs are used to write an augmented system form and a generalization of the UI observer design even for a system with an arbitrary relative degree is demonstrated. Next, the observer convergence study is presented based on the Lyapunov theory and the problem resolution will be held by using the LMI approach. Finally, illustrative examples are given to show the effectiveness of the proposed method.

\section{PReliminaries And Notations}

Throughout the paper, the following useful notations are used:

- $A^{T}$ represents the transpose of matrix $A$. 
- $A>0$ means that $\mathrm{A}$ is a symmetric positive definite matrix.

- $\rho$ represents the vector of $n_{\rho}$ known time-varying parameters which are sufficiently smooth and bounded.

- $\Theta$ : is the set of unknown parameters of dimension $n_{\theta}$, $\theta_{i} \in \Theta$ parameters to be identified $\left(i=1, \ldots, n_{\theta}\right)$.

- $I_{n}$ denotes the $n \times n$ identity matrix.

- $0_{n \times m}$ represents the $n \times m$ matrix whose entries elements are all null.

- $(.)^{\dagger}=\left[(.)^{T}(.)\right]^{-1}(.)^{T}$ is the left pseudo-inverse of the matrix (.).

We are interested by the Linear Parameter Varying (LPV) structure expressed by:

$$
\sum\left\{\begin{array}{c}
\dot{x}(t)=A(\rho) x(t)+B u(t)+F\left(y, \dot{y}, . ., y^{(n)}, \theta\right) \\
y=C x(t)
\end{array}\right.
$$

where $x(t) \in \mathbb{R}^{n}, u(t) \in \mathbb{R}^{m}, F\left(y, \dot{y}, . ., y^{(n)}, \theta\right) \in \mathbb{R}^{p}$ and $y(t) \in \mathbb{R}^{n_{y}}$ are the state vector, the input vector, the unknown part and the output of the system, respectively. $\rho$ is the vector of time-varying parameters. The matrix $A(\rho) \in \mathbb{R}^{n \times n}$ is parameter varying, the matrices $C \in \mathbb{R}^{n_{y} \times n}, B \in \mathbb{R}^{n \times m}$ are considered constant but the extension of the approach for parameter varying output matrix is straightforward.

Assumption 1: In this study, $\theta$ is the vector of constant parameters $\dot{\theta}=0$.

Definition 1: A state observer or state reconstructor converges asymptotically when the following condition $\|x(t)-\hat{x}(t)\| \rightarrow 0, t \rightarrow \infty$ holds, where $x(t)$ is the real state and $\hat{x}$ is the estimated state.

Definition 2: Kalman Criterion of observability: We say that the pair $(A, C)$ is observable when $\operatorname{rank}(\mathscr{O})=n$, with :

$$
\mathscr{O}=\left(\begin{array}{lllll}
C & C A & C A^{2} & \ldots & C A^{(n-1)}
\end{array}\right)^{T}
$$

Definition 3: Frequency Criterion of detectability : The system (1) is observable if and only if :

$$
\operatorname{rank}(\mathscr{R}(s))=n, \quad \forall s \in \mathbb{C} \text { and } \mathscr{R}(s)=\left(\begin{array}{c}
s I-A \\
C
\end{array}\right)
$$

where, $\mathscr{R}(s)$ is the Rosenbrock matrix of system $\sum(A, C)$. In practice, it is enough to verify it for the eigenvalues $s=\left(\begin{array}{llll}l_{1} & l_{1} & \ldots & l_{n}\end{array}\right)$ of the $A$ matrix.

\section{Problem Statement}

In equation (1), system's states and parameters need to be estimated. For this, we reformulate the unknown part $F$ of the model in order to test the matching condition:

Assumption 2: In equation (3), it will be assumed that $F$ can be factorized into

$$
F\left(y, \dot{y}, . ., y^{(n)}, \theta\right)=D\left(y, \dot{y}, . ., y^{(n)}\right) f(\theta)
$$

The matrix $D \in \mathbb{R}^{n \times p}$ is the measurement matrix of full column rank, i.e. $\operatorname{rank}(D)=p$. For the rest of the paper,
$D\left(y, \dot{y}, . ., y^{(n)}\right)$ is written $D(y), f(\theta)$ is the vector of unknown parameters; thus the system (1) takes the form

$$
\left\{\begin{array}{c}
\dot{x}(t)=A(\rho) x(t)+B u(t)+D(y) f(\theta) \\
y=C x(t)
\end{array}\right.
$$

The objective is to design an UI observer for LPV systems written in a TS form in order to decouple the unknown part $F\left(y, \dot{y}, \ldots, y^{(n)}, \theta\right)$, to estimate the unmeasurable state variables, then to identify parameters from the estimated unknown input.

Note that in our case, the decoupling condition is not satisfied $\operatorname{dim}(y)=n_{y}<p$. This is what we aim to recover this condition.

\section{EXACT T-S MODEL FOR NONLINEAR SYSTEMS}

The aim of this section is to find an equivalent linear form for the system in equation (3). An effective choice is the use of sector nonlinearity approach as described in [13]. We obtain a TS representation with $2^{n_{\rho}}$ sub-models.

$$
\left\{\begin{array}{c}
\dot{x}(t)=\sum_{i=1}^{n_{\rho}} \mu_{i}(\rho) A_{i} x(t)+B u(t)+D(y) f(\theta) \\
y=C x(t)
\end{array}\right.
$$

Where, the premise variables depend only on measured signals. Firstly, we suppose that the varying parameter $\rho$ is bounded within the interval $\left[\rho_{\min } \rho_{\max }\right]$.

The variables $\mu_{i}(\rho)$ are called the weighing functions, they must satisfy the following convex sum property:

$$
\left\{\begin{array}{c}
0 \leq \mu_{i}(\rho) \leq 1 \\
\sum_{i=1}^{2^{n} \rho} \mu_{i}(\rho)=1
\end{array}\right.
$$

The variables $\mu_{i}(\rho)$ are computed as follows [14]:

$$
\left\{\begin{aligned}
h_{0}^{i}(t) & =\frac{\rho_{\text {imax }}-\rho_{i}(t)}{\rho_{\text {imax }}-\rho_{\text {imin }}} \quad, \quad h_{1}^{i}(t)=\frac{\rho_{i}(t)-\rho_{\text {imin }}}{\rho_{\text {imax }}-\rho_{\text {imin }}} \\
& \Rightarrow \mu_{i}(\rho(t))=\prod_{j=1}^{n_{\rho}} h_{i j}^{i}(\rho(t))
\end{aligned}\right.
$$

\section{General Overview :}

- In the general case when $n_{y}>p$, it is clear that if the observability condition of the couple $(A(\rho), C)$ and the condition $(\operatorname{rank}(C D)=\operatorname{rank}(D))$ is verified (the output $y(t)$ has a uniform relative degree with respect to the unknown input), a state observer (UIO) for nonlinear system can be designed.

- In the case when $\left(p>n_{y}\right.$ hence $\left.\operatorname{rank}(C D) \neq \operatorname{rank}(D)\right)$, a solution is proposed to overcome this issue by considering a new structure of the UI observer and also a design procedure to identify parameters even if we have more parameters than output, as it will be shown below.

\section{OBSERVER DESIGN PROCEDURE}

Assumption 3: .

- The state $x$ is bounded (stable or stabilized),

- The pairs $\left(A_{i}, C\right)$ are observable or detectable,

- The output $y$ and its derivatives $\dot{y}_{\ldots . .} y^{(n)}$ are available.

Let us denote $\tau$ the time delay applied on the system output and $x_{a}(\mathrm{t})=\left[x, x_{\tau}\right]^{T}$ the augmented state vector, and $y_{a}(t)=\left[y, y_{\tau}\right]^{T}$ the augmented measurement vector, where 
$y_{\tau}=C x_{\tau}=y(t-\tau)$.

Remark 1: The time delay $\tau$ is carefully chosen in such a way to have signals $y$ and $y_{\tau}$ different, (the augmented $y_{a}(t)$ is of full rank and $\left.\left(p \geq n_{\theta}\right)\right)$. This can be ensured by choosing a Persistent Excitation.

Hence, the augmented model is given by state space representation as following:

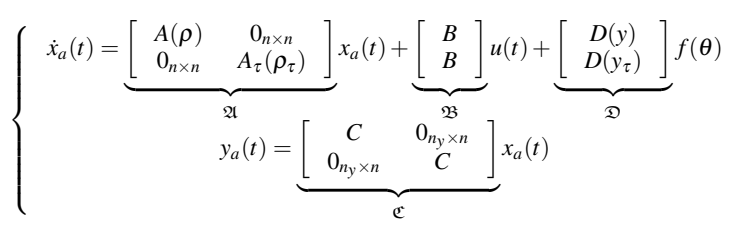

Remark 2: The matching condition for the augmented model holds: $\operatorname{rank}(\mathfrak{C D})=\operatorname{rank}\left[\begin{array}{c}C D(y) \\ C D\left(y_{\tau}\right)\end{array}\right]=\operatorname{rank}(\mathfrak{D})$.

The increase of the $\operatorname{rank}(\mathfrak{C D})$ comes from the matrix $D_{\tau}$. If the matching condition is not satisfied, the model is augmented with other delayed outputs until the condition is satisfied. In the following, and for the sake of simplicity, we expose only the case with one single delay. The general case is straightforward.

From the output equation $y_{a}=\mathfrak{C} x_{a}(t)$, the time derivative of $y_{a}(t)$ is : $\dot{y}_{a}(t)=\mathfrak{C} \mathfrak{A}(\rho) x_{a}(t)+\mathfrak{C} \mathfrak{B} u(t)+\mathfrak{C} \mathfrak{D} f(\theta)$.

The unknown part can be expressed, by model inversion, as follows :

$$
F(y, \theta)=\mathfrak{D}(\mathfrak{C} \mathfrak{D})^{\dagger}\left(\dot{y}_{a}(t)-\mathfrak{C} \mathfrak{A} x_{a}-\mathfrak{C} \mathfrak{B} u(t)\right)
$$

An observer for system (7) can be given by a dynamical system under the form:

$$
\left\{\begin{array}{c}
\dot{z}(t)=\underbrace{\left[\begin{array}{cc}
N_{1} & 0_{n \times n} \\
0_{n \times n} & N_{2}
\end{array}\right]}_{N(\rho)} z(t)+\underbrace{\left[\begin{array}{cc}
L_{1} & 0_{n \times n_{y}} \\
0_{n \times n_{y}} & L_{2}
\end{array}\right]}_{\hat{x}(t)} y_{a}(t)+\underbrace{\left[\begin{array}{c}
G_{1} \\
G_{2}
\end{array}\right]}_{G(\rho)} u(t) \\
\hat{x}_{a}(t)=\left[\begin{array}{c}
\hat{x}(\rho) \\
\hat{x}_{\tau}(t)
\end{array}\right]=z(t)-\underbrace{\left[\begin{array}{cc}
H_{1} & 0_{n \times n_{y}} \\
n_{n \times n_{y}} & H_{2}
\end{array}\right]}_{H(y)} y_{a}(t)
\end{array}\right.
$$

where matrices $N, L, G$ are to be determined to guarantee a minimal bounded of the estimation error $e=x_{a}-\hat{x}_{a}$. On the other hand, the observer gains $H$ are computed to ensure that the estimation error converges asymptotically to zero. In equation (8), $N(\rho)$ and $L(\rho)$ have the same quasi-LPV form as the matrix $\mathfrak{A}(\rho)$ and can be written in a TS form as follows:

$$
N(\rho)=\sum_{i=1}^{r_{1}} \mu_{i}(\rho) N_{i}, L(\rho)=\sum_{i=1}^{r_{1}} \mu_{i}(\rho) L_{i}, G(\rho)=\sum_{i=1}^{r_{1}} \mu_{i}(\rho) G_{i}, H(y)=\sum_{i=1}^{r_{2}} \mu_{i}(y) H_{i} \text { (9) }
$$

where, $r_{1}=2^{n_{\rho}}, r_{2}=2^{n_{y}}$

According to equations (7) and (8), the state estimation error is given by:

$$
e=x_{a}-\hat{x}_{a}=\underbrace{(I+H(y) \mathfrak{C})}_{P(y(t))} x_{a}-z
$$

From equations (7), (8) and 10), the dynamics of the state estimation error is expressed by the following differential equation:

$$
\begin{aligned}
\dot{e}(t) & =N e(t)+(\dot{P}+P \mathfrak{A}-N P-L \mathfrak{C}) x_{a}(t)+P \mathfrak{D} f(\theta) \\
& +(P \mathfrak{B}-G) u(t)
\end{aligned}
$$

Under the conditions:

$$
\begin{aligned}
\dot{P}+P \mathfrak{A}-N P-L \mathfrak{C} & =0 \\
P \mathfrak{D} & =0 \\
P \mathfrak{B}-G & =0
\end{aligned}
$$

The estimation error dynamics will be reduced to:

$$
\dot{e}(t)=N e(t)
$$

in which, $N(\rho)$ must be Hurwitz.

Equations (12) to (14) form a set of conditions to be resolved to compute the observer gains. To achieve this, the following steps in the design approach are followed. We replace the matrix $P$ in the equality (12) which leads to:

$$
\begin{aligned}
\dot{P}+P \mathfrak{A}-N P-L \mathfrak{C}=0 & \Leftrightarrow \dot{P}+P \mathfrak{A}-N-(N H+L) \mathfrak{C}=0 \\
& \Leftrightarrow \underbrace{\dot{P}+P \mathfrak{A}}_{\Gamma}-N-(\underbrace{N H+L}_{K}) \mathfrak{C}=0 \\
& \Leftrightarrow N=\Gamma-K \mathfrak{C}
\end{aligned}
$$

$\Gamma$ and $K$ are function of the parameters varying signals $\rho(t)$ and/or the measurement output $y(t)$. Then the sector nonlinear approach is used to transform the problem into a (TS) form where, $r=2^{\left(n_{\rho}+n_{y}\right)}$.

$$
\left\{\begin{array}{c}
\Gamma(\rho, y)=\dot{P}+P \mathfrak{A}=\sum_{i=1}^{r} \mu_{i}(\rho, y) \Gamma_{i} \\
K(\rho, y)=\sum_{i=1}^{r} \mu_{i}(\rho, y) K_{i}
\end{array}\right.
$$

The dynamics of the state estimation error's become:

$$
\dot{e}(t)=(\Gamma-K \mathfrak{C}) e(t)
$$

In this paper, the stability analysis of the system (7) is studied in order to obtain the observer gains that satisfy LMI conditions. This analysis is performed using the Lyapunov theorem, by considering the following Lyapunov function:

$$
V(e(t))=e(t)^{T} Q e(t), \quad Q=Q^{T}>0
$$

By using the error dynamics in equation (18), the time derivative of the lyapunov function can be written by the following expression:

$$
\dot{V}(t)=e(t)^{T}\left(N^{T} Q+Q N\right) e(t)
$$

where, $N=\sum_{i=1}^{r} \mu_{i}(\rho, y)\left(\Gamma_{i}-K_{i} \mathfrak{C}\right)$.

Using the convex sum property of the weighting functions and from (20), sufficient BMI conditions ensuring asymptotic stability are obtained as follows :

$\left(\Gamma_{i}-K_{i} \mathfrak{C}\right)^{T} Q+Q\left(\Gamma_{i}-K_{i} \mathfrak{C}\right)<0, i=1, \ldots, r$

By considering $\mathfrak{R}_{i}=Q K_{i}$, the BMI condition will be equivalent to the following LMI condition:

$$
\Gamma_{i}^{T} Q+Q \Gamma_{i}-\mathfrak{C}^{T} \mathfrak{R}_{i}^{T}-\mathfrak{R}_{i} \mathfrak{C}<0, \Gamma_{i}=P_{i} A_{i}+\dot{P}_{i}
$$

where, $P_{i}=I+H_{i} \mathfrak{C}, \dot{P}_{i}=\left(\frac{d P}{d y}\right)_{i} \times \dot{y}_{i}$

Theorem 1: The state estimation error converges asymptotically toward zero if there exist a symmetric positive definite matrix $Q \in \mathbb{R}^{n \times n}$ and a matrix $\mathfrak{R} \in \mathbb{R}^{n \times n y}$ such that the following linear matrix inequality holds

$$
\Gamma_{i}^{T} Q_{i}+Q_{i} \Gamma_{i}-\mathfrak{R}_{i} \mathfrak{C}_{i}-\mathfrak{C}_{i}^{T} \mathfrak{R}_{i}^{T}<0, \mathfrak{R}_{i}=Q K_{i}
$$


which can be solved easily by using the Yalmip toolbox (see [12] for more details).

When the state estimation error $e(t)$ converges toward zero, we have $\hat{x}(t) \rightarrow x(t)$, then the following UI estimation $\hat{F}$ is obtained by the following equation :

$$
\hat{F}(y, \theta)=\mathfrak{D}(\mathfrak{C D})^{\dagger}\left(\dot{y}_{a}(t)-\mathfrak{C A} \hat{x}_{a}(t)-\mathfrak{C} \mathfrak{B} u(t)\right)
$$

in which, the convergence of $\hat{f}$ toward $f$ can be analyzed by defining the unknown input estimation error

$$
e_{F}(t)=F(y, \theta)-\hat{F}(y, \theta)=-\mathfrak{D}(\mathfrak{C D})^{\dagger} \mathfrak{C} A e(t)
$$

knowing that $e(t)$ converges asymptotically to zero, then $e_{F}(t)$ also converges asymptotically to zero.

The UI observer design procedure is summarized by the following steps:

1) The observability condition of the couple $(\mathfrak{A}, \mathfrak{C})$ is verified following definition 2 and the rank condition $(\operatorname{rank}(\mathfrak{C D})=\operatorname{rank}(\mathfrak{D}))$ is satisfied

2) The LMI problem includes the following condition to compute the matrices $H$ as follows:

$$
\left\{\begin{array}{c}
\left(I+H_{i} \mathfrak{C}\right) \mathfrak{D}_{i}=0 \Rightarrow H_{i}=-\mathfrak{D}_{i}\left(\mathfrak{C} \mathfrak{D}_{i}\right)^{\dagger}, G_{i}=\left(I+H_{i} \mathfrak{C}\right) \mathfrak{B} \\
P_{i}=I+H_{i} \mathfrak{C} \Rightarrow \Gamma_{i}=P_{i} A_{i}+\dot{P}_{i}
\end{array}\right.
$$

3) Find gain matrices $Q$ and $\mathfrak{R}_{i}$ satisfying LMI equation (21) to ensure asymptotic convergence toward zero of the state estimation (definition 1).

4) The gains of the observer $N, L$ and $H$ are computed as follows:

$$
\left\{\begin{array}{c}
K_{i}=Q^{-1} \mathfrak{R}_{i} \\
N_{i}=\Gamma_{i}-K_{i} \mathfrak{C} \\
P_{i}=I+H_{i} \mathfrak{C} \\
L_{i}=K_{i}-N_{i} H_{i}
\end{array}\right.
$$

Parameters estimation from the unknown input :

In order to estimate the parameters of the model, let us consider the following expression $F_{i}, i=1, \ldots, p$ :

$$
\hat{F}_{i}\left(y(t), \dot{y}(t), . ., y^{(n)}, \hat{\theta}\right)=D\left(y(t) \dot{y}(t), . ., y^{(n)}\right) \hat{f}_{i}(\theta)
$$

This is a linear system of $p$ equations with $n_{\theta}$ unknowns parameters, in which ( $p<n_{\theta}$, fewer equations than unknowns). An augmented system of equation which is defined from (Eq.23) with delayed values $\left(\hat{F}_{i}\right.$ and $\left.y(t) \dot{y}(t-\tau), . ., y^{(n)}\right)$, is well posed to identify parameters. The observer (8) is synthesized to ensure the stability and convergence of the unknown inputs $\left(\hat{F}_{i}(t), \hat{F}_{i}(t-\tau)\right)$ taking into account the delay on the outputs presented in the augmented model.

\section{NUMERICAL EXAMPLES AND SIMULATIONS}

In this section, the proposed method is highlighted through an academic LTI example and an LPV model.

\section{A. Academic LTI Example}

Consider the following tutorial model parameters:

$$
\left\{\begin{array}{c}
\dot{x}(t)=A x(t)+B u(t)+F(y, \theta) \\
F(y, \theta)=D(y) f(\theta) \\
y=C x(t)
\end{array}\right.
$$

The state matrices of the system are presented as

$$
\bar{A}=\left[\begin{array}{ccc}
-1 & 4 & a_{13} \\
-5 & -2 & 0 \\
-5+a_{31} & 0 & -1+a_{33}
\end{array}\right]=A+A_{\theta}
$$

$$
A=\left[\begin{array}{ccc}
-1 & 4 & 0 \\
-5 & -2 & 0 \\
-5 & 0 & -1
\end{array}\right] B=\left[\begin{array}{l}
1 \\
1 \\
1
\end{array}\right] C=\left[\begin{array}{lll}
1 & 0 & 0 \\
0 & 0 & 1
\end{array}\right]
$$

$A_{\theta}$ is the unknown part of this model, to keep the same structure studied above, we considered $A_{\theta}$ in $F(y, \theta)$ and the nominal parameters values are :

$$
a_{13}=0.5, \quad a_{31}=0.8, \quad a_{33}=-2.9
$$

$F(y, \theta)=\{\begin{array}{l}F_{1}=y_{2} \cdot a_{13} \\ F_{2}=0 \\ F_{3}=y_{1} \cdot a_{31}+y_{2} \cdot a_{33}\end{array} \Longleftrightarrow F=\underbrace{\left[\begin{array}{ccc}y_{2} & 0 & 0 \\ 0 & 0 & 0 \\ 0 & y_{1} & y_{2}\end{array}\right]}_{D(y)} \times \underbrace{\left[\begin{array}{c}a_{13} \\ a_{31} \\ a_{33}\end{array}\right]}_{f(\boldsymbol{\theta})}$

One has $\operatorname{dim}(y)=2<\operatorname{dim}(\theta)=3$. In this case, let us consider the augmented model described by equation (7), and $\tau$ is chosen on the simulation program in SimulinK MATLAB (Transport Delay block).

We consider the UI observer described by equation (8) which satisfy $\operatorname{rank}(\mathfrak{C D})=\operatorname{rank}(\mathfrak{D})$ $\left(\operatorname{dim}\left(y, y_{\tau}\right)=4>=\operatorname{dim}(\boldsymbol{\theta})\right)$, then the observer gain are described as follow:

$$
\begin{gathered}
H_{1}=H_{2}=\left[\begin{array}{cc}
-1 & 0 \\
0 & 0 \\
0 & -1
\end{array}\right], N_{1}=N_{2}=\left[\begin{array}{ccc}
-0.5 & 0 & 0 \\
-0 & -2 & 0 \\
-0 & 0 & -0.49
\end{array}\right] \\
L_{1}=L_{2}=\left[\begin{array}{cc}
0 & 0 \\
-5.0 & 0 \\
0 & 0
\end{array}\right], G_{1}=G_{2}=\left[\begin{array}{l}
0 \\
1 \\
0
\end{array}\right]
\end{gathered}
$$

The unknown part can be estimated by a simple dynamic system inversion, since the condition $\operatorname{rank}(\mathfrak{C D})=\operatorname{rank}(\mathfrak{D})$ :

$$
\hat{F}=\mathfrak{D}(\mathfrak{C} \mathfrak{D})^{\dagger}\left(\dot{y}_{a}(t)-\mathfrak{C} \mathfrak{A} x_{a}-\mathfrak{C} \mathfrak{B} u(t)\right)
$$

From the estimate $\hat{F}$ and knowing that $F$ is written as a function of the parameter vector $\hat{f}(\theta)$ and the measurement matrix $D(y): \hat{F}=D \hat{f}$ (Eq.27), one can remark in this equation, we have three parameters and two equations $(p=$ $2<n_{\theta}=3$ ), thus to estimate parameter, we augment the equations system $\left(p=n_{\theta}=3\right)$.

Then, the following system of equations is used to estimate the unknown parameters $\theta$ :

$$
\left[\begin{array}{l}
a_{13} \\
a_{31} \\
a_{33}
\end{array}\right]=\left[\begin{array}{ccc}
y_{2}(t) & 0 & 0 \\
0 & y_{1}(t) & y_{2}(t) \\
0 & y_{1}(t-\tau) & y_{2}(t-\tau)
\end{array}\right]^{-1} \times\left[\begin{array}{c}
\hat{F}_{1}(t) \\
\hat{F}_{2}(t) \\
\hat{F}_{2}(t-\tau)
\end{array}\right]
$$

Figures (1-3) shows test simulation results of a (TS-UIO) observer, synthesized under the same conditions as before, the actual variables are given with curves in red and the estimated ones with curves in blue.

Fig. (1) shows the input $(u)$, output $(y)$ and its delayed value $\left(y_{\tau}\right)$, unknown part with delay $\left(F-F_{\tau}\right)$ and the error between nominal and estimated values of unknown part.

The estimated states using the designed observer are shown in Fig. 2 and Fig. 3 shows estimated parameters. 

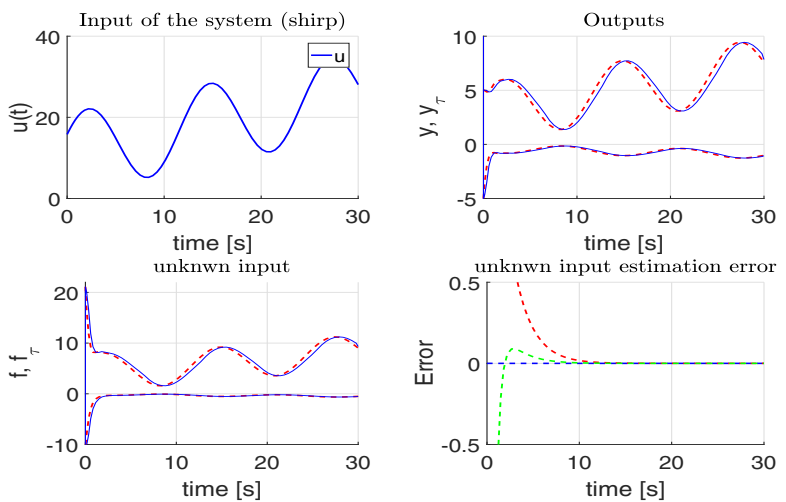

Fig. 1. Input $(u)$-output $(y)$ and its delayed value $\left(y_{\tau}\right)$-unknown input with delay $\left(F-F_{\tau}\right)$ and the error.
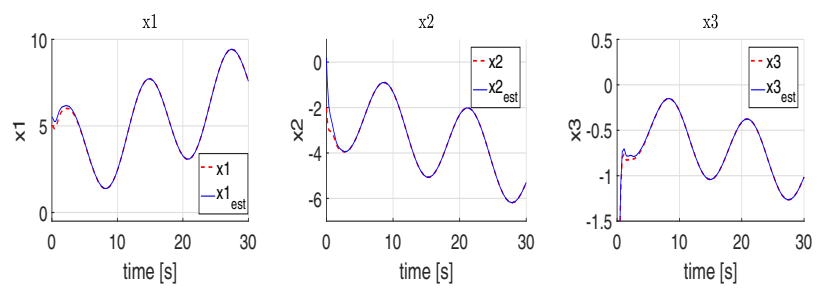

Fig. 2. States estimation
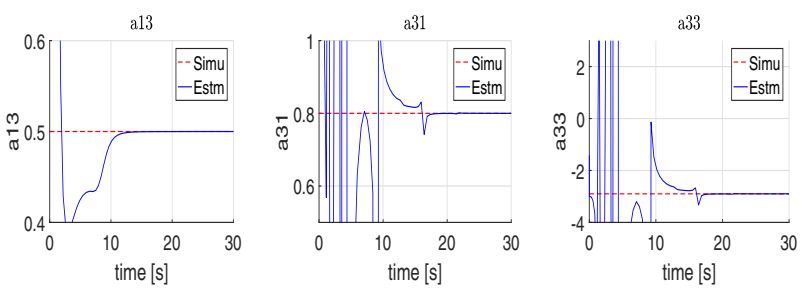

Fig. 3. Parameters estimation $a_{13}-a_{31}-a_{33}$.

Remark 3: The singularities in Fig. 3 are mainly due to the observer transient state and the conditioning of the inverse matrix of the equation (30) which sometimes passes through zero.

\section{B. Dynamic LPV descriptor Model}

Consider a continuous-time LPV descriptor system described by:

$$
\left\{\begin{aligned}
E \dot{x}(t) & =M(\rho(t)) x(t)+R(\rho) u(t)+F(y, \rho, \theta) \\
y(t) & =C x(t)
\end{aligned}\right.
$$

whereas, the matrix $M(\rho)=\left[m_{i j}\right]_{6 \times 6}, \quad R(\rho)=\left[r_{i j}\right]_{6 \times 1}$ are parameter varying, $F(y, \theta)$ is the unknown part of the model, and $C$ is the observation matrix $E=\left[e_{i j}\right]_{6 \times 6}$ is a constant nonsingular matrix, its inverse $E^{-1}$ exists. The system state matrices are :

$$
M(\rho)=\left[\begin{array}{cccccc}
0 & m_{1} \rho & 0 & 0 & 1 & 1 \\
0 & 0 & 0 & m_{2} \rho & m_{3} & m_{4} \\
0 & 0 & 0 & 1 & 0 & 0 \\
0 & m_{5} \rho & m_{6} \rho & 0 & 0 & 0 \\
m_{7} & m_{8} & m_{9} \rho & 0 & m_{10} \rho & 0 \\
m_{11} & m_{12} & m_{13} \rho & 0 & 0 & m_{14} \rho
\end{array}\right]
$$

$$
\begin{gathered}
R(\rho)=\left[\begin{array}{c}
0 \\
0 \\
0 \\
0 \\
r_{1} \rho \\
0
\end{array}\right] \quad E=\left[\begin{array}{cccccc}
e_{1} & 0 & 0 & 0 & 0 & 0 \\
0 & e_{2} & 0 & 0 & 0 & 0 \\
0 & 0 & 1 & 0 & 0 & 0 \\
e_{3} & 0 & 0 & e_{4} & 0 & 0 \\
0 & 0 & 0 & 0 & 1 & 0 \\
0 & 0 & 0 & 0 & 0 & 1
\end{array}\right] \quad C=\left[\begin{array}{cccccc}
0 & 1 & 0 & 0 & 0 & 0 \\
0 & 0 & 1 & 0 & 0 & 0 \\
0 & 0 & 0 & 0 & 1 & 0 \\
0 & 0 & 0 & 0 & 0 & 1
\end{array}\right] \\
F(\rho, y, \theta)=\left\{\begin{aligned}
F_{1} & =0 \\
F_{2} & =a_{24} \rho y_{4}+a_{25} y_{5}+a_{26} y_{6} \\
F_{3} & =0 \\
F_{4} & =a_{42} \rho y_{2}+a_{43} y_{3} \\
F_{5} & =a_{52} y_{2} \\
F_{6} & =a_{62} y_{2}
\end{aligned}\right.
\end{gathered}
$$

For further details on model parameters value $\left(e_{i j}, m_{i j}, r_{i j}\right)$ refer to Table 1.

The Linear Parameter Varying (LPV) structure is expressed by:

$$
\left\{\begin{array}{c}
\dot{x}(t)=A(\rho) x(t)+B u(t)+E^{-1} F(y, \rho, \theta) \\
y=C x(t)
\end{array}\right.
$$

$B=E^{-1} R$ and $A(\rho(t))=E^{-1} M(\rho)$ are parameter-varying matrix related to $\rho$, the unknown part can be factorized into $F(y, \theta)=D(y) f(\theta)$.

One notices that $\left.\operatorname{rank}\left(C E^{-1} D\right)\right) \neq \operatorname{rank}\left(E^{-1} D\right) \quad\left(n_{y}=4<\right.$ $\left.n_{\theta}=7\right)$, the matching condition is not fulfilled. Let us consider the augmented model described by equation (7) and the global observer described by equation (8) applied on the system (34) which satisfy $\operatorname{rank}\left(\mathfrak{C} E^{-1} \mathfrak{D}\right)=\operatorname{rank}\left(E^{-1} \mathfrak{D}\right)$ as described before.

The effectiveness of the proposed observer is illustrated with the system studied in open-loop. The input vector is presented in Fig. 5 with the varying parameters $\rho(t)$ : a measurable signal, which is used over the operating range $\rho \in\left[\begin{array}{ll}5 & 13\end{array}\right]$. The estimated states using the designed observer are shown in Fig. 5, it can be appreciated that the observer errors reach to zero.

Since asymptotic convergence, we estimate the unknown input $\hat{F}(y, \rho, \theta)$, then the unknown parameters of the model

$$
\left[\begin{array}{l}
a_{24} \\
a_{25} \\
a_{26} \\
a_{42} \\
a_{43} \\
a_{52} \\
a_{62}
\end{array}\right]=\left[\begin{array}{ccc}
\mathfrak{y}_{1} & 0_{n_{\mathfrak{y}_{1}} \times n_{\mathfrak{y}_{2}}} & 0_{n_{\mathfrak{y}_{1}}+n_{\mathfrak{y}_{3}}} \\
0_{\mathfrak{n}_{2} \times n_{\mathfrak{y}_{1}}} & \mathfrak{y}_{2} & 0_{n_{\mathfrak{y}_{2}} \times n_{\mathfrak{y}_{3}}} \\
0_{n_{\mathfrak{y}_{3}} \times n_{\mathfrak{y}_{1}}} & 0_{n_{\mathfrak{y}_{2}} \times n_{\mathfrak{y}_{3}}} & \mathfrak{y}_{3}
\end{array}\right]^{-1} \times\left[\begin{array}{c}
\hat{F}_{2}(t) \\
\hat{F}_{2}\left(t-\tau_{1}\right) \\
\hat{F}_{2}\left(t-\tau_{2}\right) \\
\hat{F}_{3}(t) \\
\hat{F}_{3}\left(t-\tau_{1}\right) \\
\hat{F}_{5}(t) \\
\hat{F}_{6}(t)
\end{array}\right]
$$

The expressions $\left(\mathfrak{y}_{1}, \mathfrak{y}_{2}, \mathfrak{y}_{3}\right)$ are as follow:

$$
\begin{gathered}
\mathfrak{y}_{1}=\left[\begin{array}{ccc}
y_{1}(t) & y_{4}(t) & y_{5}(t) \\
y_{1}\left(t-\tau_{1}\right) & y_{4}\left(t-\tau_{1}\right) & y_{5}\left(t-\tau_{1}\right) \\
y_{1}\left(t-\tau_{2}\right) & y_{4}\left(t-\tau_{2}\right) & y_{5}\left(t-\tau_{2}\right)
\end{array}\right]^{-1} \\
\mathfrak{y}_{2}=\left[\begin{array}{ccc}
y_{1}(t) & y_{2}(t) \\
y_{1}\left(t-\tau_{1}\right) & y_{2}\left(t-\tau_{1}\right)
\end{array}\right]^{-1}, \mathfrak{y}_{3}=\left[\begin{array}{cc}
y_{1}(t) & 0 \\
0 & y_{1}(t)
\end{array}\right]^{-1}
\end{gathered}
$$

Parameters estimation is done assuming that all the states are available (either measurable or estimated), we applied the delay on the outputs and the estimated unknown input to reconstruct the augmented model, the unknown vector of parameters is also well estimated from the model inversion given by equations (36-37) and depicted in Fig. 6 with the error between nominal and estimated value.
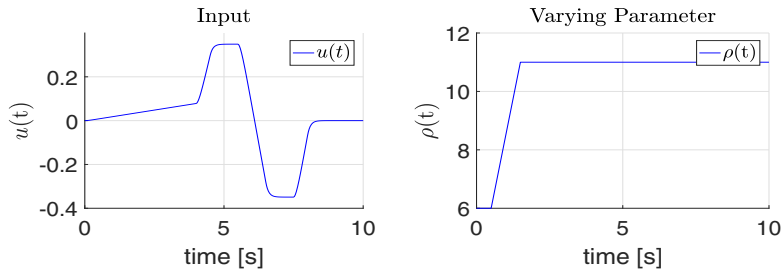

Fig. 4. (right) steering angle (left) Varying parameter 

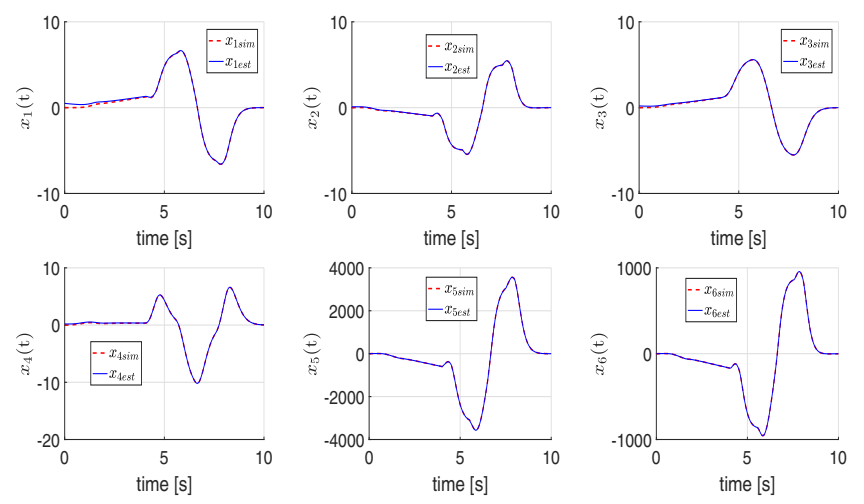

Fig. 5. States estimation.
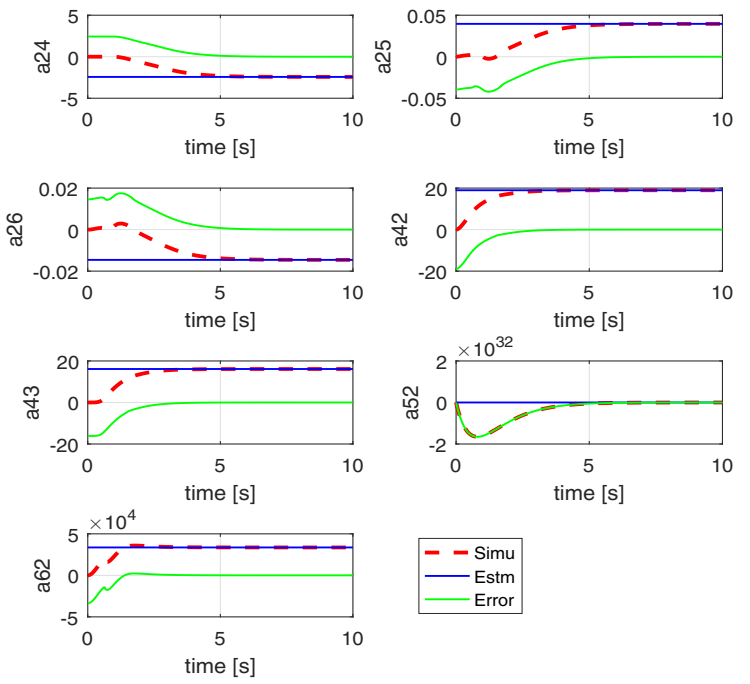

Fig. 6. Actual parameters (red) estimated parameters (blue) error (green)

\section{Result and Interpretation}

From simulations above, we can draw the following conclusions :

The proposed observer shows a good estimation accuracy and proves the reliability of the approach. It is clearly highlight that the observer converges to the actual states despite the initial conditions of the model. The states and parameters are then well estimated with minimal error in the figure (5-6).

These results show the ability of the method to estimate simultaneously the dynamic states and the unknown parameters of the model.

The vector parameters is sensitive to states and outputs variations when we use a fairly poor excitation, The estimation of these parameters, using measurement corresponding to low excitation tests, will be difficult. So, it must be ensured that the excitation makes it possible to cover all the dynamics taken into account in the model and the appropriate parameters will be well excited.
TABLE I

NUMERICAL VALUES LPV MODEL

$$
\begin{aligned}
& m_{1}=-220, m_{2}=-5.3056, m_{3}=0.9, m_{4}=-0.3, m_{5}=115, \\
& m_{6}=10^{3}, m_{7}=-92960, m_{8}=-88312, m_{9}=5976, \\
& m_{10}=-5, m_{11}=-9^{4}, m_{12}=3^{4}, m_{13}=4^{3}, m_{14}=-5 \\
& e_{1}=220, e_{2}=24, e_{3}=-110, e_{4}=67, r_{1}=98936 \\
& a_{24}=-2.43, a_{25}=0.04, a_{26}=-0.015 \\
& a_{42}=18.96, a_{43}=16.13 a_{52}=-88312, a_{62}=33616.76 \\
& \hline
\end{aligned}
$$

\section{CONCLUSION}

This paper presents an observer approach (UIO) for jointly reconstruct the state variables and the unknown parameters for a large class of nonlinear systems. An augmented unknown input observer based on delayed measurements and Takagi sugeno transformation is proposed to reconstruct state and unknown part of the model.

Sufficient conditions for the existence of the estimator are given in terms of (LMIs) to ensure the asymptotic state and parameters estimation error convergence. Using the obtained information of the unknown input estimation, the unknown parameters are well estimated.

Illustrative examples are presented to verify the effectiveness of the proposed method.

\section{REFERENCES}

[1] Luenberger, D. (1966). Observers for multivariable systems. IEEE Transactions on Automatic Control, 11(2), 190-197.

[2] Dabladji, M. E. H., Ichalal, D., Arioui, H., Mammar, S., Fridman, L. (2014). Estimation of lateral dynamics and road curvature for twowheeled vehicles: A HOSM observer approach.

[3] Bolognani, S., Tubiana, L., Zigliotto, M. (2003). Extended Kalman filter tuning in sensorless PMSM drives. IEEE Transactions on Industry Applications, 39(6), 1741-1747.

[4] S. H. Wang, E. J. Davison and P. Dorato, "Observing the states of systems with unmeasurable disturbances," IEEE Trans. Automat. Contr., vol. AC-20, pp 716-717, 1975.

[5] Darouach, M., Zasadzinski, M., Xu, S. J. (1994). Full-order observers for linear systems with unknown inputs. IEEE transactions on automatic control, 39(3), 606-609.

[6] D. Koenig. Unknown input proportional multiple-integral observer design for linear descriptor systems: Application for state and fault estimation. IEEE Trans. Automat. Contr. vol 50, pp. 212-217, 2005.

[7] In Besanon, G. (2007)a Nonlinear observers and applications (Vol 363, pp. 1-33). Berlin: springer.

[8] Briat, C. (2014). LPV Time-Delay SystemsAnalysis, Observation, Filtering Control.

[9] T.Takagi and M. Sugeno. Fuzzy identification of systems and its applications to modeling and control. IEEE Transactions on Systems, Man, and Cybernetics vol.15, pp. 116-132, 1985.

[10] K. Tanaka, T. Ikeda, and H. Wang, Fuzzy regulators and fuzzy observers, Relaxed stability conditions and lmi-based designs, IEEE Transactions on Fuzzy Systems, vol. 6, no. 2, pp. 250-265, 1998.

[11] Kuhlmann, L. (2015) Parameter and state estimation of nonlinear systems using a multi-observer under the supervisory framework. IEEE Transactions on Automatic Control, 60(9), 2336-2349.

[12] Ichalal, D., Marx, B., Ragot, J., Maquin, D. (2009, June). Simultaneous state and unknown inputs estimation with PI and PMI observers for Takagi Sugeno model with unmeasurable premise variables.

[13] Tanaka, K., Wang, H. O. (2004). Fuzzy control systems design and analysis: a linear matrix inequality approach. John Wiley Sons.

[14] Morère, Y. (2001). Mise en oeuvre de lois de commande pour les modèles flous de type Takagi-Sugeno (Doctoral dissertation, Valenciennes).

[15] https://fr.mathworks.com/help/simulink/slref/transportdelay.html 